\title{
Quantization of the Lie Bialgebra of String Topology
}

\author{
Xiaojun Chen ${ }^{1}$, Farkhod Eshmatov ${ }^{1}$, Wee Liang Gan ${ }^{2}$ \\ 1 Department of Mathematics, University of Michigan, Ann Arbor, MI 48109, U.S.A. \\ E-mail: xch@umich.edu; eshmatov@umich.edu \\ 2 Department of Mathematics, University of California, Riverside, CA 92521, U.S.A. \\ E-mail: wlgan@math.ucr.edu
}

Received: 13 March 2009 / Accepted: 18 July 2010

Published online: 7 October 2010 - (C) The Author(s) 2010. This article is published with open access at Springerlink.com

\begin{abstract}
Let $M$ be a smooth, simply-connected, closed oriented manifold, and $L M$ the free loop space of $M$. Using a Poincaré duality model for $M$, we show that the reduced equivariant homology of $L M$ has the structure of a Lie bialgebra, and we construct a Hopf algebra which quantizes the Lie bialgebra.
\end{abstract}

\section{Introduction}

Let $M$ be a smooth, simply-connected, closed oriented manifold, and $L M$ the free loop space of $M$. In this paper, by the reduced equivariant homology of $L M$, we mean the $S^{1}$-equivariant homology of $L M$ relative to a point which is a constant loop in $M$. We give an algebraic chain model of a Lie bialgebra structure on the reduced equivariant homology of the free loop space $L M$, and we construct a quantization of the Lie bialgebra. Chas and Sullivan have shown in [9] that the $S^{1}$-equivariant homology of $L M$ relative to the constant loops has the natural structure of a Lie bialgebra. Our constructions are compatible with the one which they gave.

The motivations for the constructions in this paper are as follows. In [15], Goldman showed that the free homotopy classes of closed curves on a Riemann surface $\mathcal{S}$ form a Lie algebra; and subsequently in [24], Turaev proved that they in fact form a Lie bialgebra. Moreover, Turaev showed that the Lie bialgebra admits a quantization, which can be realized as the skein algebra of links in $\mathcal{S} \times I$, where $I$ is the unit interval. In [8] and [9], Chas and Sullivan generalized the Lie bialgebra of Goldman and Turaev to the higher dimensional case, and initiated the subject of string topology. Algebraic chain models for string topology have been studied by many authors. In particular, Felix and Thomas [12] used a Poincaré duality model of the manifold $M$ that was constructed by Lambrechts and Stanley [20] to give a chain model of the BV-algebra structure on the singular homology of $L M$ (see also [17]).

On the other hand, there is a quiver analogue of the Lie bialgebra of Goldman and Turaev. This is the necklace Lie bialgebra studied by V. Ginzburg [14] and T. Schedler 
[23]. Schedler constructed a quantization of the necklace Lie bialgebra in analogy with Turaev's quantization. In this paper, we show that the Poincaré duality model of $M$ can also be used to construct a chain model of a Lie bialgebra structure on the reduced equivariant homology of $L M$. Furthermore, a quantization of the Lie bialgebra can be constructed following Schedler's method.

We will prove the following theorem (cf. Theorems 5, 9 and 15 of this paper):

Theorem 1. (i) Let $V$ be a counital coaugmented simply-connected DG open Frobenius algebra over a field $\mathbb{k}$ of characteristic zero. Then there is a natural involutive Lie bialgebra structure on the reduced cyclic homology of $V$, and a Hopf algebra over $\mathbb{k}[h]$ (where $h$ is a formal parameter) which quantizes this Lie bialgebra.

(ii) Given a smooth, closed, oriented and simply connected manifold $M$, there is a counital coaugmented simply-connected DG open Frobenius algebra $V$ over $\mathbb{Q}$ which models the chain complex of $M$, and the reduced cyclic homology of $V$ is isomorphic to the reduced equivariant homology of $L M$. Therefore, the reduced equivariant homology of LM has a Lie bialgebra structure, and the Hopf algebra of (i) quantizes this Lie bialgebra.

The definition of a DG open Frobenius algebra will be given later in Definition 3. By cyclic homology, we mean the homology of the Connes' complex for a coalgebra (see Definition 2). It would be interesting to find a geometric interpretation of the Hopf algebra in Theorem 1, similar to Turaev's construction. The construction in Theorem 1 (ii) of the DG open Frobenius algebra of a manifold is due to Lambrechts and Stanley (see [20]).

In [6], Cattaneo, Frölhich and Pedrini gave a topological field theoretic interpretation of string topology. In particular, they showed that under some mild conditions of the gauge group, the Poisson bracket of the generalized Wilson loops, when applied to two equivariant homology classes in $L M$, is the same as the generalized Wilson loop applied to the Lie bracket of two equivariant homology classes. They also suggested several approaches of quantizing this field theory (see $[6, \S 8.3]$ ). We expect that the Hopf algebra given by Theorem 1 will appear in the associated quantum field theory, which they conjectured to be related to algebraic structures on the Vassiliev homology of links in higher dimensional manifolds.

Another interesting problem is the existence of natural representations of the string topology Lie bialgebra and its quantization. In the Riemann surface case, Goldman ([15]) showed that there is a Lie algebra homomorphism from the homotopy classes of curves to the smooth functions on the moduli space of flat connections on the surface. Similar results in the quiver case have been obtained by Ginzburg ([14]) and Schedler ([23]). In the string topology case, a Lie algebra homomorphism from the equivariant homology to the smooth functions on some related moduli space has been studied by Abbaspour, Zeinalian, and Tradler (see [1] and [2]). Besides Turaev, the quantization of the Lie algebra of curves has also been studied by Andersen, Mattes and Reshetikhin $([3,4])$ from the Vassiliev knot theory point of view. We hope to address this problem in the future and relate it to the work of Cattaneo and Rossi (see [7] and references therein).

The rest of the paper is devoted to the proof of Theorem 1. In Sect. 2 we recall a chain complex model for the free loop space of manifolds, due independently to K.-T. Chen [10] and J.D.S. Jones [19]. From this, one can construct a chain model for the reduced equivariant homology of the free loop space. In Sect. 3 we construct the Lie bialgebra structure on the reduced equivariant homology of the free loop space, and in the last section, Sect. 4, we construct the Hopf algebra which quantizes the Lie bialgebra, 
following the work of Schedler. Our main computation is contained in the proof of Lemma 14.

\section{Reduced Equivariant Homology}

2.1. Cyclic homology of coalgebras. First, let us recall the definitions of Hochschild homology and cyclic homology of a coalgebra.

Let $(C, d)$ be a DG coalgebra over a field $\mathbb{k}$ of characteristic 0 . For any element $a \in C$, denote the coproduct of $a$ by $\sum_{(a)} a^{\prime} \otimes a^{\prime \prime}$. We shall write $C$ [1] for $C$ with degrees of elements shifted down by 1 .

Definition 2. (i) The Hochschild chain complex of $C$, denoted by $\left(\operatorname{Hoch}_{*}(C), b\right)$, is the vector space $\prod_{n=0}^{\infty} C \otimes C[1]^{\otimes n}$ with the differential $b$ defined on homogeneous elements by:

$$
\begin{aligned}
b\left(a_{0},\right. & \left.a_{1}, \ldots, a_{n}\right) \\
:= & -\sum_{i=0}^{n}(-1)^{\varepsilon_{i-1}}\left(a_{0}, \ldots, d a_{i}, \ldots, a_{n}\right) \\
& +\sum_{i=0}^{n} \sum_{\left(a_{i}\right)}(-1)^{\varepsilon_{i-1}+\left|a_{i}^{\prime}\right|-1}\left(a_{0}, \ldots, a_{i}^{\prime}, a_{i}^{\prime \prime}, \ldots, a_{n}\right) \\
& +\sum_{\left(a_{0}\right)}(-1)^{\left(\left|a_{0}^{\prime}\right|-1\right)\left(\varepsilon_{n}-\left|a_{0}^{\prime}\right|\right)}\left(a_{0}^{\prime \prime}, a_{1}, \ldots, a_{n}, a_{0}^{\prime}\right),
\end{aligned}
$$

where $\varepsilon_{i}=\left|a_{0}\right|+\cdots+\left|a_{i}\right|-i$. The associated homology is called the Hochschild homology, and is denoted by $\mathrm{HH}_{*}(C)$.

(ii) For any $\alpha=\left(a_{0}, \ldots, a_{n}\right) \in \operatorname{Hoch}_{*}(C)$, define

$$
t(\alpha):=(-1)^{\left(\left|a_{0}\right|-1\right)\left(\varepsilon_{n}-\left|a_{0}\right|\right)}\left(a_{1}, \ldots, a_{n}, a_{0}\right),
$$

and let $N=i d+t+\cdots+t^{n}$. The image of $N$, denoted by $\mathrm{CC}_{*}(C)$, is a subcomplex of $\operatorname{Hoch}_{*}(C)$, called the Connes complex. The homology of $\mathrm{CC}_{*}(C)$ is called the cyclic homology of $C$, and is denoted by $\mathrm{HC}_{*}(C)$.

In the above definition of a Hochschild chain complex, we call (2) the internal differential, and (3)+(4) the external differential. The Hochschild homology of a coalgebra is also known as the coHochschild homology (see [18]).

2.2. Poincaré duality model. Throughout this paper, we assume that $M$ is a simplyconnected, compact, oriented smooth manifold of dimension $\mathrm{m}$. Denote by $L M$ the free loop space of $M$. There is a natural $S^{1}$-action on $L M$. We shall take $\mathbb{k}$ to be the field of rational numbers, and write $C_{*}(-)$ and $C^{*}(-)$ for the singular chain complex and the singular cochain complex, respectively. We grade $C^{*}(-)$ negatively.

The chain complex $C_{*}(M)$ has a partially defined product given by intersection of transversal chains, and a coproduct given by the Alexander-Whitney approximation of the diagonal embedding $M \hookrightarrow M \times M$. In fact, $C_{*}(M)$ is a partially defined (noncommutative) DG open Frobenius algebra over $\mathbb{Z}$; however, over $\mathbb{Q}$, one may define the Frobenius algebra structure fully, due to a result by P. Lambrechts and D. Stanley [20]: 
Definition 3 (Open Frobenius algebra). Let $V$ be a DG vector space over $\mathbb{k}$. A DG open Frobenius algebra (of degree $\mathrm{m})$ on $V$ is the triple $(V, \cdot, \Delta)$ such that:

(i) $(V, \cdot)$ is a commutative DG algebra (whose product is of degree $-\mathrm{m})$;

(ii) $(V, \Delta)$ is a cocommutative DG coalgebra;

(iii) The following identity, called the module compatibility, holds: for any $a, b \in V$,

$$
\Delta(a \cdot b)=\sum(-1)^{\mathrm{m}\left|a^{\prime}\right|} a^{\prime} \otimes a^{\prime \prime} \cdot b=\sum a \cdot b^{\prime} \otimes b^{\prime \prime},
$$

where $\Delta a=\sum a^{\prime} \otimes a^{\prime \prime}$, and $\Delta b=\sum a^{\prime} \otimes b^{\prime \prime}$.

Theorem 4 (Lambrechts and Stanley). There is a finite dimensional commutative DG algebra $A$ such that $A$ is simply-connected, $A$ is quasi-isomorphic to $C^{*}(M)$, and there is an A-bimodule isomorphism of degree $\mathrm{m}$ from $A$ to its dual $A^{\vee}$ that induces the Poincaré duality isomorphism $H^{*}(M) \rightarrow H_{*+\mathrm{m}}(M)$ on homology.

Proof. This is immediate from applying [20, Theorem 1.1] to the Sullivan minimal model of $M$.

The DG algebra $A$ in Theorem 4 is called a Poincaré duality model for $M$. In [20], the construction of the isomorphism from $A$ to $A^{\vee}$ depends on the (possibly degenerate) pairing on the differential forms (and hence on the minimal model) of $M$, and at present we are not able to show any functoriality property of $A$ (see also [20, Sect.7]). For the rest of this section, we let $V=A^{\vee}$, where $A$ is a Poincaré duality model. Thus, $V$ is a cocommutative DG coalgebra. The linear isomorphism from $A$ to $V[\mathrm{~m}]$ induces the structure of a commutative DG algebra on $V$ whose product has degree $-\mathrm{m}$. Moreover, the coproduct is a morphism of $V$-bimodules. Therefore, $V$ is a DG open Frobenius algebra which is simply-connected, has a counit $\varepsilon: V \rightarrow \mathbb{k}$, and a coaugmentation $\eta: \mathbb{k} \hookrightarrow V$. Let $C=\operatorname{Coker}(\eta)$, the coaugmentation coideal of $V$. The homology of $\mathrm{CC}_{*}(C)$ is called the reduced cyclic homology of $V$.

The following theorem is essentially due to Jones [19].

Theorem 5. The reduced equivariant chain complex of $L M$ is quasi-isomorphic to $\mathrm{CC}_{*}(C)[1]$.

Proof. The Hochschild complex $\operatorname{Hoch}_{*}(V)$ is quasi-isomorphic to the normalized Hochschild complex $\prod_{n=0}^{\infty} V \otimes C[1]^{\otimes n}$ whose differential $b$ is given by the same formula (1). For the rest of this proof, we shall denote by $\operatorname{Hoch}_{*}(V)$ the normalized Hochschild complex of $V$. Then Connes' operator $B$ is defined by

$$
\begin{aligned}
B: \operatorname{Hoch}_{*}(V) & \longrightarrow \operatorname{Hoch}_{*+1}(V) \\
\left(a_{0}, a_{1}, \ldots, a_{n}\right) & \longmapsto \sum_{i=1}^{n} \varepsilon\left(a_{0}\right)\left(a_{i}, \ldots, a_{n}, a_{1}, \ldots, a_{i-1}\right) .
\end{aligned}
$$

One has $B^{2}=0, b \circ B+B \circ b=0$. Let $u$ be a formal variable of degree 2, and define the differential

$$
b+u^{-1} B: \operatorname{Hoch}_{*}(V)[u] \rightarrow \operatorname{Hoch}_{*}(V)[u]
$$

by

$$
\left(b+u^{-1} B\right)\left(\alpha \otimes u^{n}\right)= \begin{cases}b(\alpha) \otimes u^{n}+B(\alpha) \otimes u^{n-1}, & \text { if } n>0 \\ b(\alpha), & \text { if } n=0\end{cases}
$$


Since $A$ is quasi-isomorphic to $C^{*}(M)$, it follows by a well-known result of Jones (see [19, Theorem A] or [11, Theorem 1.5.1, Corollary 1.5.2]) that the equivariant chain complex $C_{*}^{S^{1}}(L M)$ is quasi-isomorphic to $\left(\operatorname{Hoch}_{*}(V)[u], b+u^{-1} B\right)$. Let

$$
\overline{\operatorname{Hoch}}_{*}(V)=C \oplus \prod_{n=1}^{\infty} V \otimes C[1]^{\otimes n}
$$

be the reduced Hochschild complex. Then the reduced equivariant chain complex of $L M$ and $\left(\overline{\operatorname{Hoch}}_{*}(V)[u], b+u^{-1} B\right)$ are quasi-isomorphic. On the other hand, note that the normalized and reduced Hochschild complexes are non-negatively graded, so the bicomplex $\left(\overline{\operatorname{Hoch}}_{*}(V)[u], b+u^{-1} B\right)$ lies in the first quadrant. It follows by a standard argument using filtrations (see [21, Proposition 2.2.14]) that the map

$$
\begin{aligned}
& \left(\overline{\operatorname{Hoch}}_{*}(V)[u], b+u^{-1} B\right) \rightarrow C C_{*}(C)[1] \\
& \alpha \otimes u^{n} \mapsto \begin{cases}B(\alpha), & \text { if } n=0, \\
0, & \text { if } n>0,\end{cases}
\end{aligned}
$$

is a quasi-isomorphism. The gives the desired result.

Remark 6. The equivariant homology of $L M$ is isomorphic to the direct sum of the reduced equivariant homology of $L M$ with $\mathbb{k}[u]($ where $\operatorname{deg}(u)=2)$.

Since $V$ is simply-connected, we may replace the direct product in the definition of $\mathrm{Hoch}_{*}(C)$ by direct sum.

\section{Lie Bialgebra}

3.1. Construction of the Lie bialgebra. In this section, $C$ is the coaugmentation coideal of a counital coaugmented simply-connected DG open Frobenius algebra $V$ (of degree $\mathrm{m})$. One can also take $C$ to be $V$ itself. We shall write \pm for signs determined by the usual Koszul convention.

Definition 7 (Lie coalgebra). Let $L$ be a vector space over $\mathbb{k}$. A skew-symmetric map $\delta: L \rightarrow L \otimes L$ defines a Lie coalgebra structure on $L$ if

$$
\left(\tau^{2}+\tau+i d\right) \circ(\delta \otimes i d) \circ \delta=0: L \rightarrow L \otimes L \otimes L,
$$

where $\tau$ is the permutation $a \otimes b \otimes c \mapsto \pm c \otimes a \otimes b$, for $a, b, c \in$ L. The map $\delta$ is called the cobracket and (6) is called the co-Jacobi identity.

Definition 8 (Lie bialgebra). Suppose $(L,\{\}$,$) is a Lie algebra and (L, \delta)$ is a Lie coalgebra. The triple $(L,\{\},, \delta)$ defines a Lie bialgebra on $L$ if the following identity, called the Drinfeld compatibility, holds:

$$
\delta\{a, b\}=\{a, \delta(b)\}+\{\delta(a), b\} .
$$

If moreover, $\{,\} \circ \delta: L \rightarrow L$ vanishes identically, the Lie bialgebra $(L,\{\},, \delta)$ is called involutive. 
Let $L:=\left(\mathrm{CC}_{*}(C)[1]\right)[\mathrm{m}-2]=\mathrm{CC}_{*}(C)[\mathrm{m}-1]$, where $\mathrm{CC}_{*}(C)$ is defined in the previous section. We shall write elements of $L$ in the form $N\left(\left[a_{1}|\cdots| a_{n}\right]\right)$, where $a_{i} \in C[1]$ for $i=1, \ldots, n$.

Define on $L$ the following two operators: $\{\}:, L \otimes L \rightarrow L$ by

$$
\{\alpha, \beta\}:=\sum_{i, j} \pm \varepsilon\left(a_{i} \cdot b_{j}\right) N\left(\left[a_{i+1}|\cdots| a_{n}\left|a_{1}\right| \cdots\left|a_{i-1}\right| b_{j+1}|\cdots| b_{m}\left|b_{1}\right| \cdots \mid b_{j-1}\right]\right)
$$

and $\delta: L \rightarrow L \otimes L$ by

$$
\delta(\alpha):=\sum_{i<j} \pm \varepsilon\left(a_{i} \cdot a_{j}\right) N\left(\left[a_{1}|\cdots| a_{i-1}\left|a_{j+1}\right| \cdots \mid a_{n}\right]\right) \wedge N\left(\left[a_{i+1}|\cdots| a_{j-1}\right]\right)
$$

for any homogeneous $\alpha=N\left(\left[a_{1}|\cdots| a_{n}\right]\right), \beta=N\left(\left[b_{1}|\cdots| b_{m}\right]\right) \in L$, where in the above $\varepsilon$ is the augmentation, and in (9), $a \wedge b$ means $a \otimes b-b \otimes a$, and will also be written as $a \otimes b-$ Alt.

Theorem 9. Let $L$ be as above. Then $(L,\{\},, \delta)$ forms an involutive DG Lie bialgebra.

The Lie bracket $\{$,$\} is of degree 0$, and the Lie cobracket $\delta$ is of degree $2(2-\mathrm{m})$. The rest of this section is devoted to the proof of Theorem 9. The proof is divided into several steps.

3.2. Proof of the DG Lie algebra. The product on $V$ is graded commutative, hence if we shift the degree of $C$ down by 1 , the induced pairing $\varepsilon(a \cdot b): C[1] \otimes C[1] \rightarrow \mathbb{k}$ is graded skew-symmetric. Therefore the bracket $\{$,$\} defined by (8) is graded skew-symmetric.$ We now show the Jacobi identity: for any $\alpha=N\left(\left[a_{1}|\cdots| a_{n}\right]\right), \beta=N\left(\left[b_{1}|\cdots| b_{m}\right]\right)$, $\gamma=N\left(\left[c_{1}|\cdots| c_{p}\right]\right) \in L$,

$$
\begin{aligned}
& \{\{\alpha, \beta\}, \gamma\} \\
& \quad=\sum_{i, j, k, l} \pm \varepsilon\left(a_{i} b_{j}\right) \varepsilon\left(a_{k} c_{l}\right) N\left(\left[a_{1}|\cdots| b_{j+1}|\cdots| b_{j-1}|\cdots| c_{l+1}|\cdots| c_{l-1}|\cdots| a_{n}\right]\right) \\
& \quad+\sum_{i, j, k, l} \pm \varepsilon\left(a_{i} b_{j}\right) \varepsilon\left(b_{k} c_{l}\right) N\left(\left[a_{1}|\cdots| b_{j+1}|\cdots| c_{l+1}|\cdots| c_{l-1}|\cdots| b_{j-1}|\cdots| a_{n}\right]\right)
\end{aligned}
$$

Similarly, we have

$$
\begin{aligned}
& \{\{\beta, \gamma\}, \alpha\} \\
& =\sum_{i, j, k, l} \pm \varepsilon\left(b_{j} c_{l}\right) \varepsilon\left(b_{k} a_{i}\right) N\left(\left[b_{1}|\cdots| c_{l+1}|\cdots| c_{l-1}|\cdots| a_{i+1}|\cdots| a_{i-1}|\cdots| b_{m}\right]\right) \\
& \quad+\sum_{i, j, k, l} \pm \varepsilon\left(b_{j} c_{l}\right) \varepsilon\left(c_{k} a_{i}\right) N\left(\left[b_{1}|\cdots| c_{l+1}|\cdots| a_{i+1}|\cdots| a_{i-1}|\cdots| c_{l-1}|\cdots| b_{m}\right]\right),
\end{aligned}
$$


and

$$
\begin{aligned}
& \{\{\alpha, \beta\}, \gamma\} \\
& =\sum_{i, j, k, l} \pm \varepsilon\left(c_{l} a_{i}\right) \varepsilon\left(c_{k} b_{j}\right) N\left(\left[c_{1}|\cdots| a_{i+1}|\cdots| a_{i-1}|\cdots| b_{j+1}|\cdots| b_{j-1}|\cdots| c_{p}\right]\right) \\
& \quad+\sum_{i, j, k, l} \pm \varepsilon\left(c_{l} a_{i}\right) \varepsilon\left(a_{k} b_{j}\right) N\left(\left[c_{1}|\cdots| a_{i+1}|\cdots| b_{j+1}|\cdots| b_{j-1}|\cdots| a_{i-1}|\cdots| c_{p}\right]\right) .
\end{aligned}
$$

Note that by the cyclic invariance of $N$, (10) cancels with (15), so do (11) with (12) and (13) with (14). This proves the Jacobi identity.

We next show that $b$ respects the bracket. It is easy to see that the bracket thus defined commutes with the internal differential, hence we only check that it commutes with the external differential. For any $\alpha=N\left(\left[a_{1}|\cdots| a_{n}\right]\right), \beta=N\left(\left[b_{1}|\cdots| b_{m}\right]\right)$,

$b(\alpha)=\sum_{i=1}^{n} N\left(\left[a_{1}|\cdots| a_{i}^{\prime}\left|a_{i}^{\prime \prime}\right| \cdots \mid a_{n}\right]\right), \quad$ and $\quad b(\beta)=\sum_{j=1}^{n} N\left(\left[b_{1}|\cdots| b_{j}^{\prime}\left|b_{j}^{\prime \prime}\right| \cdots \mid b_{m}\right]\right)$.

Therefore,

$$
\begin{aligned}
& \{b(\alpha), \beta\} \\
& =\sum_{i, k, l} \pm \varepsilon\left(a_{k} b_{l}\right) N\left(\left[a_{k+1}|\cdots| a_{n}\left|a_{1}\right| \cdots\left|a_{i}^{\prime}\right| a_{i}^{\prime \prime}|\cdots| a_{k-1}\left|b_{l+1}\right| \cdots\left|b_{m}\right| b_{1}|\cdots| b_{l-1}\right]\right) \\
& \quad+\sum_{i, l} \pm \varepsilon\left(a_{i}^{\prime} b_{l}\right) N\left(\left[a_{i}^{\prime \prime}|\cdots| a_{n}\left|a_{1}\right| \cdots\left|a_{i-1}\right| b_{l+1}|\cdots| b_{m}\left|b_{1}\right| \cdots \mid b_{l-1}\right]\right) \\
& \quad+\sum_{i, l} \pm \varepsilon\left(a_{i}^{\prime \prime} b_{l}\right) N\left(\left[a_{i+1}|\cdots| a_{n}\left|a_{1}\right| \cdots\left|a_{i-1}\right| a_{i}^{\prime}\left|b_{l+1}\right| \cdots\left|b_{m}\right| b_{1}|\cdots| b_{l-1}\right]\right)
\end{aligned}
$$

$\{\alpha, b(\beta)\}$

$$
\begin{aligned}
= & \sum_{j, k, l} \pm \varepsilon\left(a_{k} b_{l}\right) N\left(\left[a_{k+1}|\cdots| a_{n}\left|a_{1}\right| \cdots\left|a_{k-1}\right| b_{l+1}|\cdots| b_{j}^{\prime}\left|b_{j}^{\prime \prime}\right| \cdots\left|b_{m}\right| b_{1}|\cdots| b_{l-1}\right]\right) \\
& +\sum_{k, j} \pm \varepsilon\left(a_{k} b_{j}^{\prime}\right) N\left(\left[a_{k+1}|\cdots| a_{n}\left|a_{1}\right| \cdots\left|a_{k-1}\right| b_{j}^{\prime \prime}|\cdots| b_{m}\left|b_{1}\right| \cdots \mid b_{j-1}\right]\right) \\
& +\sum_{k, j} \pm \varepsilon\left(a_{k} b_{j}^{\prime \prime}\right) N\left(\left[a_{k+1}|\cdots| a_{n}\left|a_{1}\right| \cdots\left|a_{k-1}\right| b_{j+1}|\cdots| b_{m}\left|b_{1}\right| \cdots\left|b_{j-1}\right| b_{j}^{\prime}\right]\right),
\end{aligned}
$$

while

$$
\begin{aligned}
& b\{\alpha, \beta\} \\
& =\sum_{i, k, l} \pm \varepsilon\left(a_{k} b_{l}\right) N\left(\left[a_{k+1}|\cdots| a_{n}\left|a_{1}\right| \cdots\left|a_{i}^{\prime}\right| a_{i}^{\prime \prime}|\cdots| a_{k-1}\left|b_{l+1}\right| \cdots\left|b_{m}\right| b_{1}|\cdots| b_{l-1}\right]\right) \\
& \quad+\sum_{j, k, l} \pm \varepsilon\left(a_{k} b_{l}\right) N\left(\left[a_{k+1}|\cdots| a_{n}\left|a_{1}\right| \cdots\left|a_{k-1}\right| b_{l+1}|\cdots| b_{j}^{\prime}\left|b_{j}^{\prime \prime}\right| \cdots\left|b_{m}\right| b_{1}|\cdots| b_{l-1}\right]\right) .
\end{aligned}
$$


Note that (17) and (21) cancel, so do (18) and (20). The remaining terms of $\{b(\alpha), \beta\}+$ $\{\alpha, b(\beta)\}$ are identical to $(22)+(23)$, which is exactly $b\{\alpha, \beta\}$.

3.3. Proof of the DG Lie coalgebra. The cobracket is skew-symmetric. The co-Jacobi identity holds due by a similar computation as the Jacobi identity, and so we leave its verification to the reader.

Next, we show that $b$ respects the cobracket. As before, we check that the external differential commutes with the cobracket: by definition,

$$
\begin{aligned}
& \delta N\left(\left[a_{1}|\cdots| a_{n}\right]\right) \\
& \quad=\sum_{i<j} \pm \varepsilon\left(a_{i} a_{j}\right) N\left(\left[a_{1}|\cdots| a_{i-1}\left|a_{j+1}\right| \cdots \mid a_{n}\right]\right) \otimes N\left(\left[a_{i+1}|\cdots| a_{j-1}\right]\right)-\text { Alt }
\end{aligned}
$$

hence

$$
\begin{aligned}
b( & \delta N\left(\left[a_{1}|\cdots| a_{n}\right]\right) \\
= & \sum_{i<j, k} \pm \varepsilon\left(a_{i} a_{j}\right) N\left(\left[a_{1}|\cdots| a_{k}^{\prime}\left|a_{k}^{\prime \prime}\right| \cdots\left|a_{i-1}\right| a_{j+1}|\cdots| a_{n}\right]\right) \\
& \otimes N\left(\left[a_{i+1}|\cdots| a_{j-1}\right]\right)-\mathrm{Alt} \\
& +\sum_{i<j, l} \pm \varepsilon\left(a_{i} a_{j}\right) N\left(\left[a_{1}|\cdots| a_{i-1}\left|a_{j+1}\right| \cdots \mid a_{n}\right]\right) \\
& \otimes N\left(\left[a_{i+1}|\cdots| a_{l}^{\prime}\left|a_{l}^{\prime \prime}\right| \cdots \mid a_{j-1}\right]\right)-\mathrm{Alt},
\end{aligned}
$$

while $\delta \sum_{k} N\left(\left[a_{1}|\cdots| a_{k}^{\prime}\left|a_{k}^{\prime \prime}\right| \cdots \mid a_{n}\right]\right)$ has not only (24) and (25), but also

$$
\begin{aligned}
& \left.\sum_{i, k} \pm \varepsilon\left(a_{i} a_{k}^{\prime}\right) N\left(a_{1}|\cdots| a_{i-1}\left|a_{k}^{\prime \prime}\right| \cdots \mid a_{n}\right]\right) \\
& \quad \otimes N\left(\left[a_{i+1}|\cdots| a_{k-1}\right]\right)-\text { Alt } \\
& \quad+\sum_{i, k} \pm \varepsilon\left(a_{i} a_{k}^{\prime \prime}\right) N\left(\left[a_{1}|\cdots| a_{i-1}\left|a_{k+1}\right| \cdots \mid a_{n}\right]\right) \\
& \quad \otimes N\left(\left[a_{i+1}|\cdots| a_{k-1} \mid a_{k}^{\prime}\right]\right)-\text { Alt } \\
& \quad+\sum_{k, j} \pm \varepsilon\left(a_{i}^{\prime} a_{j}\right) N\left(\left[a_{1}|\cdots| a_{k-1}\left|a_{j+1}\right| \cdots \mid a_{n}\right]\right) \\
& \quad \otimes N\left(\left[a_{k}^{\prime \prime}\left|\alpha_{k+1}\right| \cdots \mid a_{j-1}\right]\right)-\text { Alt } \\
& \quad+\sum_{k, j} \pm \varepsilon\left(a_{k}^{\prime \prime} a_{j}\right) N\left(\left[a_{1}|\cdots| a_{k}^{\prime}\left|a_{j+1}\right| \cdots \mid a_{n}\right]\right) \\
& \quad \otimes N\left(\left[a_{k+1}|\cdots| a_{j-1}\right]\right)-\text { Alt. }
\end{aligned}
$$

Since $C$ is an open Frobenius algebra, by the module compatibility (5), (26) cancels with (29), and (27) cancels with (28), and hence $b$ commutes with the cobracket.

3.4. Proof of the Drinfeld compatibility. Let $\alpha=N\left(\left[a_{1}|\cdots| a_{n}\right]\right)$ and $\beta=$ $N\left(\left[b_{1}|\cdots| b_{m}\right]\right)$, and write $\delta(\alpha)=\alpha^{(1)} \otimes \alpha^{(2)}$ and $\delta(\beta)=\beta^{(1)} \otimes \beta^{(2)}$. 
We have

$$
\{\alpha, \beta\}=\sum_{i, j} \pm \varepsilon\left(a_{i} b_{j}\right) N\left(\left[a_{i+1}|\cdots| a_{i-1}\left|b_{j+1}\right| \cdots \mid b_{j-1}\right]\right)
$$

and

$$
\begin{aligned}
\delta\{\alpha & \beta\} \\
= & \sum_{i, j, k, l} \pm \varepsilon\left(a_{i} b_{j}\right) \varepsilon\left(a_{k} a_{l}\right) N\left(\left[a_{k+1}|\cdots| a_{l-1}\right]\right) \\
& \otimes N\left(\left[a_{l+1}|\cdots| a_{i-1}\left|b_{j+1}\right| \cdots\left|b_{j-1}\right| a_{i+1}|\cdots| a_{k-1}\right]\right) \\
& +\sum_{i, j, k, l} \pm \varepsilon\left(a_{i} b_{j}\right) \varepsilon\left(a_{k} b_{l}\right) N\left(\left[a_{k+1}|\cdots| a_{i-1}\left|b_{j+1}\right| \cdots \mid b_{l-1}\right]\right) \\
& \otimes N\left(\left[b_{l+1}|\cdots| b_{j-1}\left|a_{i+1}\right| \cdots \mid a_{k-1}\right]\right) \\
& +\sum_{i, j, k, l} \pm \varepsilon\left(a_{i} b_{j}\right) \varepsilon\left(a_{l} a_{k}\right) N\left(\left[a_{k+1}|\cdots| a_{i-1}\left|b_{j+1}\right| \cdots\left|b_{j-1}\right| a_{i+1}|\cdots| a_{l-1}\right]\right) \\
& \otimes N\left(\left[a_{l+1}|\cdots| a_{k-1}\right]\right) \\
& +\sum_{i, j, k, l} \pm \varepsilon\left(a_{i} b_{j}\right) \varepsilon\left(b_{k} b_{l}\right) N\left(\left[b_{k+1}|\cdots| b_{l-1}\right]\right) \\
& \otimes N\left(\left[b_{l+1}|\cdots| b_{j-1}\left|a_{i+1}\right| \cdots\left|a_{i-1}\right| b_{j+1}|\cdots| b_{k-1}\right]\right) \\
& +\sum_{i, j, k, l} \pm \varepsilon\left(a_{i} b_{j}\right) \varepsilon\left(b_{k} a_{l}\right) N\left(\left[b_{k+1}|\cdots| b_{j-1}\left|a_{i+1}\right| \cdots \mid a_{l-1}\right]\right) \\
& \otimes N\left(\left[a_{l+1}|\cdots| a_{i-1}\left|b_{j+1}\right| \cdots \mid b_{k-1}\right]\right) \\
& +\sum_{i, j, k, l} \pm \varepsilon\left(a_{i} b_{j}\right) \varepsilon\left(b_{l} b_{k}\right) N\left(\left[b_{k+1}|\cdots| b_{j-1}\left|a_{i+1}\right| \cdots\left|a_{i-1}\right| b_{j+1}|\cdots| b_{l-1}\right]\right) \\
& \otimes N\left(\left[b_{l+1}|\cdots| b_{k-1}\right]\right) .
\end{aligned}
$$

In the above, the second summation and the fifth summation cancel with each other. The first summation is equal to $\alpha^{(1)} \otimes\left\{\alpha^{(2)}, \beta\right\}$; the third summation is equal to $\left\{\alpha^{(1)}, \beta\right\} \otimes \alpha^{(2)}$; the fourth summation is equal to $\beta^{(1)} \otimes\left\{\alpha, \beta^{(2)}\right\}$; and the sixth summation is equal to $\left\{\alpha, \beta^{(1)}\right\} \otimes \beta^{(2)}$. Thus we obtain the Drinfeld compatibility.

3.5. Proof of the involutivity. Let $\alpha=N\left(\left[a_{1}|\cdots| a_{n}\right]\right)$, then

$$
\begin{aligned}
\delta(\alpha)= & \sum_{i<j} \pm \varepsilon\left(a_{i} a_{j}\right) N\left(\left[a_{1}|\cdots| a_{i-1}\left|a_{j+1}\right| \cdots \mid a_{n}\right]\right) \otimes N\left(\left[a_{i+1}|\cdots| a_{j-1}\right]\right) \\
& -\sum_{i<j} \pm \varepsilon\left(a_{i} a_{j}\right) N\left(\left[a_{i+1}|\cdots| a_{j-1}\right]\right) \otimes N\left(\left[a_{1}|\cdots| a_{i-1}\left|a_{j+1}\right| \cdots \mid a_{n}\right]\right) .
\end{aligned}
$$

By a similar argument as above, one checks that $\{,\} \circ \delta=0$ holds identically.

The above constructions and proofs, except for the compatibilities of the differential with the Lie bracket and cobracket, are similar to the proof of the Lie bialgebras of Turaev [24], Chas-Sullivan [9], Hamilton [16] and Schedler [23]. 


\section{Quantization of the Lie Bialgebra}

4.1. Construction of the Hopf algebra. In this section, we construct a DG Hopf algebra which quantizes the DG Lie bialgebra of Sect. 3. We follow Schedler [23] closely. We will also define a new differential in Definition 12 below which is not present in [23].

Definition 10 (Quantization). Let $h$ be a formal parameter. Suppose A is a Hopf algebra over $\mathbb{k}[h]$. We say A quantizes the Lie bialgebra $(L,\{\},, \delta)$ if there is a Hopf algebra isomorphism

$$
\phi: A / h A \stackrel{\cong}{\longrightarrow} U(L),
$$

where $U(L)$ is the universal enveloping algebra of $L$, such that for any $x_{0} \in L$, and any $x \in A, \phi(x)=x_{0}$,

$$
\frac{1}{h}\left(\Delta(x)-\Delta^{\mathrm{op}}(x)\right) \equiv \delta\left(x_{0}\right) \quad \bmod h
$$

where $\Delta^{\mathrm{op}}$ is the opposite comultiplication of $A$.

Definition 11. Let $C H:=C \otimes_{\mathbb{k}} \mathbb{k}\left[\mu, \mu^{-1}\right]$, where $\mu$ is a formal variable (of degree 0 ). We shall write an element $a \otimes \mu^{u} \in C H$ as $(a, u)$ and call $u \in \mathbb{Z}$ the height of $(a, u)$.

Let $\widehat{\operatorname{Hoch}}_{*}(C H)$ be the graded vector space $\bigoplus_{n=0}^{\infty} C H \otimes C H[1]^{\otimes n}$, and denote by $\widehat{\mathrm{CC}}_{*}(C H)$ the subspace of cyclically invariant elements in $\widehat{\operatorname{Hoch}}_{*}(C H)$. Let

$$
L H:=\left(\widehat{C C}_{*}(C H)[1]\right)[\mathrm{m}-2]=\widehat{C C}_{*}(C H)[\mathrm{m}-1] .
$$

There is a canonical projection $L H \rightarrow L$ by forgetting the heights in $L H$ (recall $L$ is given in §3.1). Let $S L H$ be the symmetric algebra of $L H$.

Definition 12. Define a differential b on SLH so that on the homogeneous components it is given by

$$
\begin{aligned}
b(N( & {\left.\left.\left[\left(a_{1,1}, h_{1,1}\right)|\cdots|\left(a_{1, p_{1}}, h_{1, p_{1}}\right)\right]\right) \bullet \cdots \bullet N\left(\left[\left(a_{n, 1}, h_{n, 1}\right)|\cdots|\left(a_{n, p_{n}}, h_{n, p_{n}}\right)\right]\right)\right) } \\
:= & -\sum_{i=1}^{n} \sum_{j=1}^{p_{i}} \pm \cdots \bullet N\left(\left[\left(a_{i, 1}, h_{i, 1}\right)|\cdots|\left(d a_{i, j}, h_{i, j}\right)|\cdots|\left(a_{i, p_{i}}, h_{i, p_{i}}\right)\right]\right) \bullet \cdots \\
& +\sum_{i=1}^{n} \sum_{j=1}^{p_{i}} \sum_{\left(a_{(i, j)}\right)} \pm N\left(\left[\left(a_{1,1}, \widehat{h}_{1,1}\right)|\cdots|\left(a_{1, p_{1}}, \widehat{h}_{1, p_{1}}\right)\right]\right) \bullet \cdots \\
& \quad \cdots \bullet N\left(\left[\left(a_{i, 1}, \widehat{h}_{i, 1}\right)|\cdots|\left(a_{i, j}^{\prime}, h_{i, j}\right)\left|\left(a_{i, j}^{\prime \prime}, h_{i, j}+1\right)\right| \cdots \mid\left(a_{i, p_{i}}, \widehat{h}_{i, p_{i}}\right)\right]\right) \bullet \cdots
\end{aligned}
$$

where in (32) and (33), for all $\left(i^{\prime}, j^{\prime}\right) \neq(i, j)$,

$$
\widehat{h}_{i^{\prime}, j^{\prime}}= \begin{cases}h_{i^{\prime}, j^{\prime}} & \text { if } h_{i^{\prime}, j^{\prime}} \leq h_{i, j} \\ h_{i^{\prime}, j^{\prime}}+1 & \text { if } h_{i^{\prime}, j^{\prime}}>h_{i, j} .\end{cases}
$$


Remark 13. In the above definition, we assign $a_{i, j}^{\prime}$ and $a_{i, j}^{\prime \prime}$ with heights $h_{i, j}$ and $h_{i, j}+1$ respectively, and raise all heights $h_{i^{\prime}, j^{\prime}}$ greater than $h_{i, j}$ to $h_{i, j}+1$. The coassociativity of $C$ implies that $b^{2}=0$.

Let $h$ be a formal parameter of degree $2(\mathrm{~m}-2)$. The differential $b$ on $S L H$ extends to a differential on the $\mathbb{k}[h]$-module $S L H[h]$. Consider the subcomplex of $S L H[h]$ which is spanned by elements whose homogeneous components are of the form:

$$
\left.N\left[\left(a_{1,1}, h_{1,1}\right)|\cdots|\left(a_{1, p_{1}}, h_{1, p_{1}}\right)\right]\right) \bullet \cdots \bullet N\left(\left[\left(a_{k, 1}, h_{k, 1}\right)|\cdots|\left(a_{k, p_{k}}, h_{k, p_{k}}\right)\right]\right),
$$

where all the $h_{i, j}$ are distinct. Denote this subcomplex by $\widetilde{S L H}[h]$.

Let $\tilde{A}$ be the quotient module of $\widetilde{S L H}[h]$ defined by identifying any element of the form (35) with other elements obtained by replacing $h_{i, j}$ with any $\tilde{h}_{i, j}$ satisfying $h_{i, j}<h_{i^{\prime}, j^{\prime}}$ if and only if $\tilde{h}_{i, j}<\tilde{h}_{i^{\prime}, j^{\prime}}$. Let $\tilde{B}$ be the submodule of $\tilde{A}$ generated by elements of the following form:

$$
\begin{array}{ll}
X-X_{i, j, i^{\prime}, j^{\prime}}^{\prime}-X_{i, j, i^{\prime}, j^{\prime}}^{\prime \prime}, & \text { where } i \neq i^{\prime}, h_{i, j}<h_{i^{\prime}, j^{\prime}}, \\
& \text { and } \nexists\left(i^{\prime \prime}, j^{\prime \prime}\right) \text { with } h_{i, j}<h_{i^{\prime \prime}, j^{\prime \prime}}<h_{i^{\prime}, j^{\prime}} ; \\
X-X_{i, j, i, j^{\prime}}^{\prime}-h X_{i, j, i, j^{\prime}}^{\prime \prime}, & \text { where } h_{i, j}<h_{i, j^{\prime}}, \\
& \text { and } \nexists\left(i^{\prime \prime}, j^{\prime \prime}\right) \text { with } h_{i, j}<h_{i^{\prime \prime}, j^{\prime \prime}}<h_{i, j^{\prime}},
\end{array}
$$

where the $X^{\prime}$ and $X^{\prime \prime}$ terms are defined as follows: if $i \neq i^{\prime}, X_{i, j, i^{\prime}, j^{\prime}}^{\prime}$ is the same as $X$ except that the heights $h_{i, j}$ and $h_{i^{\prime}, j^{\prime}}$ are interchanged, while $X_{i, j, i^{\prime}, j^{\prime}}^{\prime \prime}$ replaces the components $N\left(\left[\left(a_{i, 1}, h_{i, 1}\right)|\cdots|\left(a_{i, p_{i}}, h_{i, p_{i}}\right)\right]\right)$ and $N\left(\left[\left(a_{i^{\prime}, 1}, h_{i^{\prime}, 1}\right)|\cdots|\left(a_{i^{\prime}, p_{i^{\prime}}}, h_{i^{\prime}, p_{i^{\prime}}}\right)\right]\right)$ by

$$
\begin{aligned}
& \pm \\
& \quad \varepsilon\left(a_{i, j} a_{i^{\prime}, j^{\prime}}\right) N\left(\left[\left(a_{i, j+1}, h_{i, j+1}\right)|\cdots|\left(a_{i, j-1}, h_{i, j-1}\right) \mid\left(a_{i^{\prime}, j^{\prime}+1}, h_{i^{\prime}, j^{\prime}+1}\right)\right.\right. \\
& \left.\left.\quad \times|\cdots|\left(a_{i^{\prime}, j^{\prime}-1}, h_{i^{\prime}, j^{\prime}-1}\right)\right]\right) ;
\end{aligned}
$$

similarly, $X_{i, j, i, j^{\prime}}^{\prime}$ is the same as $X$ but with the heights $h_{i, j}$ and $h_{i, j^{\prime}}$ interchanged, while $X_{i, j, i, j^{\prime}}^{\prime \prime}$ is given by replacing the component with the following two components:

$$
\begin{aligned}
& \pm \varepsilon\left(a_{i, j} a_{i, j^{\prime}}\right) N\left(\left[\left(a_{i, j^{\prime}+1}, h_{i, j^{\prime}+1}\right)|\cdots|\left(a_{i, j-1}, h_{i, j-1}\right)\right]\right) \bullet N\left(\left[\left(a_{i, j+1}, h_{i, j+1}\right)\right.\right. \\
& \left.\left.\quad \times|\cdots|\left(a_{i, j^{\prime}-1}, h_{i, j^{\prime}-1}\right)\right]\right) .
\end{aligned}
$$

Lemma 14. Let $\tilde{A}$ and $\tilde{B}$ be as above. Then $\tilde{A}$ is a chain complex and $\tilde{B}$ is a subcomplex of $\tilde{A}$.

Proof. It is clear that $b$ is well-defined on $\tilde{A}$, and so $\tilde{A}$ is a chain complex. We have to check that $\tilde{B}$ is a subcomplex of $\tilde{A}$.

The equivalence relation (36) only involves operations on two components in the elements of $\widetilde{S L H}[h]$, so without loss of generality, we may assume

$$
X=N\left(\left[\left(a_{1}, 2 h_{1}\right)|\cdots|\left(a_{n}, 2 h_{n}\right)\right]\right) \bullet N\left(\left[\left(b_{1}, 2 g_{1}\right)|\cdots|\left(b_{m}, 2 g_{m}\right)\right]\right) .
$$

Suppose that in $X$, the heights $2 h_{i}$ and $2 g_{j}$ satisfy condition (36). Then

$$
\begin{aligned}
X- & X^{\prime}-X^{\prime \prime} \\
= & \pm N\left(\left[\left(a_{1}, 2 h_{1}\right)|\cdots|\left(a_{n}, 2 h_{n}\right)\right]\right) \bullet N\left(\left[\left(b_{1}, 2 g_{1}\right)|\cdots|\left(b_{m}, 2 g_{m}\right)\right]\right) \\
& \mp N\left(\left[\left(a_{1}, 2 h_{1}\right)|\cdots|\left(a_{i}, 2 g_{j}\right)|\cdots|\left(a_{n}, 2 h_{n}\right)\right]\right) \bullet N\left(\left[\left(b_{1}, 2 g_{1}\right)\right.\right. \\
& \left.\left.\quad \times|\cdots|\left(b_{j}, 2 h_{i}\right)|\cdots|\left(b_{m}, 2 g_{m}\right)\right]\right) \\
& \mp \varepsilon\left(a_{i} b_{j}\right) N\left(\left[\left(a_{i+1}, 2 h_{i+1}\right)|\cdots|\left(a_{i-1}, 2 h_{i-1}\right)\left|\left(b_{j+1}, 2 g_{j+1}\right)\right| \cdots \mid\left(b_{j-1}, 2 g_{j-1}\right)\right]\right) .
\end{aligned}
$$


Therefore

$$
\begin{aligned}
b(X)= & \sum_{k \neq i} \pm N\left(\left[\cdots\left|\left(a_{k}^{\prime}, 2 h_{k}\right)\right|\left(a_{k}^{\prime \prime}, 2 h_{k}+1\right) \mid \cdots\right]\right) \otimes N\left(\left[\cdots\left|\left(b_{l}, 2 g_{l}\right)\right| \cdots\right]\right) \\
& +\sum \pm N\left(\left[\cdots\left|\left(a_{i}^{\prime}, 2 h_{i}\right)\right|\left(a_{i}^{\prime \prime}, 2 h_{i}+1\right) \mid \cdots\right]\right) \otimes N\left(\left[\cdots\left|\left(b_{l}, 2 g_{l}\right)\right| \cdots\right]\right) \\
& +\sum_{l \neq j} \pm N\left(\left[\cdots\left|\left(a_{k}, 2 h_{k}\right)\right| \cdots\right]\right) \otimes N\left(\left[\cdots\left|\left(b_{l}^{\prime}, 2 g_{l}\right)\right|\left(b_{l}^{\prime \prime}, 2 g_{l}+1\right) \mid \cdots\right]\right) \\
& +\sum_{i} \pm N\left(\left[\cdots\left|\left(a_{k}, 2 h_{k}\right)\right| \cdots\right] \otimes N\left(\left[\cdots\left|\left(b_{j}^{\prime}, 2 g_{j}\right)\right|\left(b_{j}^{\prime \prime}, 2 g_{j}+1\right) \mid \cdots\right]\right)\right. \\
b\left(X^{\prime}\right)= & \sum_{k \neq i} \pm N\left(\left[\cdots\left|\left(a_{k}^{\prime}, 2 h_{k}\right)\right|\left(a_{k}^{\prime \prime}, 2 h_{k}+1\right) \mid \cdots\right]\right) \otimes N\left(\left[\cdots\left|\left(b_{j}, 2 h_{i}\right)\right| \cdots\right]\right) \\
& +\sum_{l} \pm N\left(\left[\cdots\left|\left(a_{i}^{\prime}, 2 g_{j}\right)\right|\left(a_{i}^{\prime \prime}, 2 g_{j}+1\right) \mid \cdots\right]\right) \otimes N\left(\left[\left(\cdots\left|\left(b_{j}, 2 h_{i}\right)\right| \cdots\right)\right]\right) \\
& +\sum_{l \neq j} \pm N\left(\left[\cdots\left|\left(a_{i}, 2 g_{j}\right)\right| \cdots\right]\right) \otimes N\left(\left[\cdots\left|\left(b_{l}^{\prime}, 2 g_{l}\right)\right|\left(b_{l}^{\prime \prime}, 2 g_{l}+1\right) \mid \cdots\right]\right) \\
& +\sum_{1 \neq N} \pm N\left(\left[\cdots\left|\left(a_{i}, 2 g_{j}\right)\right| \cdots\right]\right) \otimes N\left(\left[\cdots\left|\left(b_{j}^{\prime}, 2 h_{i}\right)\right|\left(b_{j}^{\prime \prime}, 2 h_{i}+1\right) \mid \cdots\right]\right) \\
b\left(X^{\prime \prime}\right)= & \sum_{k \neq i} \pm \varepsilon\left(a_{i} b_{j}\right) N\left(\left[\cdots\left|\left(a_{k}^{\prime}, 2 h_{k}\right)\right|\left(a_{k}^{\prime \prime}, 2 h_{k}+1\right) \mid \cdots\right]\right) \\
& +\sum_{l \neq j} \pm \varepsilon\left(a_{i} b_{j}\right) N\left(\left[\cdots\left|\left(b_{l}^{\prime}, 2 g_{l}\right)\right|\left(b_{l}^{\prime \prime}, 2 g_{l}+1\right) \mid \cdots\right]\right)
\end{aligned}
$$

It is plain that both $(38)-(42)-(46)$ and $(40)-(44)-(47)$ are contained in $\tilde{B}$. To see that $(39)+(41)-(43)-(45)$ is also contained in $\tilde{B}$, we introduce the following interpolating terms:

$$
\begin{aligned}
& \sum \pm N\left(\left[\cdots\left|\left(a_{i}^{\prime}, 2 h_{i}\right)\right|\left(a_{i}^{\prime \prime}, 2 g_{j}\right) \mid \cdots\right]\right) \otimes N\left(\left[\cdots\left|\left(b_{j}, 2 h_{i}+1\right)\right| \cdots\right]\right) \\
& \sum \pm \varepsilon\left(a_{i}^{\prime \prime} b_{j}\right) N\left(\left[\left(a_{i+1}, 2 h_{i+1}\right)|\cdots|\left(a_{i}^{\prime}, 2 h_{i}\right)\left|\left(b_{j+1}, 2 g_{j+1}\right)\right| \cdots \mid\left(b_{j-1}, 2 g_{j-1}\right)\right]\right), \\
& \sum \pm N\left(\left[\cdots\left|\left(a_{i}^{\prime}, 2 h_{i}\right)\right|\left(a_{i}^{\prime \prime}, 2 g_{j}+1\right) \mid \cdots\right]\right) \otimes N\left(\left[\cdots\left|\left(b_{j}, 2 g_{j}\right)\right| \cdots\right]\right) \\
& \sum \pm \varepsilon\left(a_{i}^{\prime} b_{j}\right) N\left(\left[\left(a_{i}^{\prime \prime}, 2 g_{j}+1\right)|\cdots|\left(a_{i-1}, 2 h_{i-1}\right)\left|\left(b_{j+1}, 2 g_{j+1}\right)\right| \cdots \mid\left(b_{j-1}, 2 g_{j-1}\right)\right]\right) \\
& \sum \pm N\left(\left[\cdots\left|\left(a_{i}, 2 g_{j}\right)\right| \cdots\right]\right) \otimes N\left(\left[\cdots\left|\left(b_{j}^{\prime}, 2 h_{i}\right)\right|\left(b_{j}^{\prime \prime}, 2 g_{j}+1\right) \mid \cdots\right]\right) \\
& \sum \pm \varepsilon\left(a_{i} b_{j}^{\prime}\right) N\left(\left[\left(a_{i+1}, 2 h_{i+1}\right)|\cdots|\left(a_{i-1}, 2 h_{i-1}\right)\left|\left(b_{j}^{\prime \prime}, 2 g_{j}+1\right)\right| \cdots \mid\left(b_{j-1}, 2 g_{j-1}\right)\right]\right) \\
& \sum \pm N\left(\left[\cdots\left|\left(a_{i}, 2 h_{i}+1\right)\right| \cdots\right]\right) \otimes N\left(\left[\cdots\left|\left(b_{j}^{\prime}, 2 h_{i}\right)\right|\left(b_{j}^{\prime \prime}, 2 g_{j}\right) \mid \cdots\right]\right) \\
& \sum \pm \varepsilon\left(a_{i} b_{j}^{\prime \prime}\right) N\left(\left[\left(a_{i+1}, 2 h_{i+1}\right)|\cdots|\left(a_{i-1}, 2 h_{i-1}\right)\left|\left(b_{j+1}, 2 g_{j+1}\right)\right| \cdots \mid\left(b_{j}^{\prime}, 2 h_{i}\right)\right]\right)
\end{aligned}
$$

One has

$$
\begin{array}{ll}
(39)-(48)-(49) \in \tilde{B}, & (41)-(52)-(53) \in \tilde{B} \\
(50)-(43)-(51) \in \tilde{B}, & (54)-(45)-(55) \in \tilde{B} .
\end{array}
$$


Moreover,

$$
(48)=(50), \quad(52)=(54), \quad(49)=-(53), \quad(51)=-(55) .
$$

Hence, $(39)+(41)-(43)-(45) \in \tilde{B}$.

By a similar argument, the subspace spanned by elements of the form $X-X^{\prime}-h X^{\prime \prime}$ in (37) is also stable under $b$, and therefore $\tilde{B}$ is a subcomplex of $\tilde{A}$.

Theorem 15. Let $A=\tilde{A} / \tilde{B}$. There is a DG Hopf algebra structure on A, which quantizes the DG Lie bialgebra $(L,\{\},, \delta)$ of Theorem 9. Moreover, A is isomorphic to $U(L)[h]$ as $\mathbb{k}[h]$-modules.

The proof of the above theorem is given in the following subsections.

4.2. Proof of DG algebra. For any two elements $X, X^{\prime} \in A$, define the product of $X$ and $X^{\prime}$ as follows: suppose $X, X^{\prime}$ are both represented by elements of the form (35); let $X^{\prime \prime}$ be the element which is the same as $X^{\prime}$ but with the corresponding heights $h_{i^{\prime}, j^{\prime}}\left(X^{\prime}\right)$ replaced by $h_{i^{\prime}, j^{\prime}}\left(X^{\prime}\right)+C$, where $C=1+\max _{i, j, i^{\prime}, j^{\prime}}\left(h_{i, j}(X)-h_{i^{\prime}, j^{\prime}}\left(X^{\prime}\right)\right)$. Here, $h_{i, j}(X)$ are the heights in $X$ and similarly $h_{i^{\prime}, j^{\prime}}\left(X^{\prime}\right)$ are the heights in $X^{\prime}$. Thus, $X^{\prime \prime}$ is obtained from $X^{\prime}$ by shifting the heights of the latter such that its heights are larger than those of $X$. The product of $X$ and $X^{\prime}$ is defined to be $X \bullet X^{\prime \prime}$. It is easy to see that this is well-defined, and commutes with the boundary $b$.

4.3. Proof of DG coalgebra. For an element $X$ in the form of (35), let

$$
P:=P_{X}:=\left\{(i, j) \mid 1 \leq i \leq k, 1 \leq j \leq p_{i}\right\} .
$$

If $(i, j) \in P$, we let

$$
(i, j)+(0,1)= \begin{cases}(i, j+1) & \text { if } j<p_{i}, \\ (i, 1) & \text { if } j=p_{i} .\end{cases}
$$

Let $n$ be an integer greater than or equal to 2. Now let $I$ be any subset of $P$ such that $\# I$ is even, and let $\phi: I \rightarrow I$ be an involutive, fixed point-free map, where by being involutive we mean $\phi^{2}=i d$. We call $(I, \phi, f)$ an $n$-labeling of $X$ if

$$
f: P \rightarrow\{1,2, \ldots, n\}
$$

is a map such that:

$$
f(i, j)= \begin{cases}f((i, j)+(0,1)), & \text { if }(i, j) \notin I ; \\ f(\phi(i, j)+(0,1)), & \text { if }(i, j) \in I,\end{cases}
$$

and

$$
f(i, j)>f(\phi(i, j)) \text { if and only if } h_{i, j}>h_{\phi(i, j)} \text {, for }(i, j) \in I \text {. }
$$

For an $n$-labeling $(I, \phi, f)$, let $q: P \rightarrow P$ be given by

$$
(i, j) \mapsto \begin{cases}(i, j)+(0,1), & \text { if }(i, j) \notin I, \\ \phi(i, j)+(0,1), & \text { otherwise, }\end{cases}
$$


and define

$$
g: P \backslash I \rightarrow P \backslash I
$$

by the following: for $(i, j) \in P \backslash I$, let $g(i, j)$ be the first element not in $I$ under the iterations of the map $q$. Since $q$ is a permutation of the finite set $P, g$ is well-defined.

Suppose the orbits of $P$ under iterations of $q$ is $\left\{Q_{1}, \ldots, Q_{w}\right\}$. Then $f$ descends to a map $\widehat{f}:\left\{Q_{1}, \ldots, Q_{w}\right\} \rightarrow\{1, \ldots, n\}$, where $\widehat{f}\left(Q_{m}\right)=f(i, j)$ for any $(i, j) \in Q_{m}$, $1 \leq m \leq w$.

Similarly, suppose the orbits of $P \backslash I$ under iterations of $g$ is $\left\{P_{1}, \ldots, P_{l}\right\}$. Then $f$ descends to a map $\bar{f}:\left\{P_{1}, \ldots, P_{l}\right\} \rightarrow\{1, \ldots, n\}$, where $\bar{f}\left(P_{m}\right)=f(i, j)$ for any $(i, j) \in P_{m}, 1 \leq m \leq l$. Suppose $P_{m}(1 \leq m \leq l)$ is the orbit of $(i, j)$ under $g$; then we define an element $X_{m} \in L H$ by

$$
X_{m}=N\left(\left[\left(a_{i, j}, h_{i, j}\right)\left|\left(a_{g(i, j)}, h_{g(i, j)}\right)\right| \cdots\right]\right) .
$$

Let $1 \leq i \leq n$. Now define an element $X_{(I, \phi, f)}^{(i)}$ in $A$ by

$$
X_{(I, \phi, f)}^{(i)}= \begin{cases}1 & \text { if } f^{-1}(i)=\emptyset, \\ 0 & \text { if } \#\left(\bar{f}^{-1}(i)\right)<\#\left(\widehat{f}^{-1}(i)\right), \\ X_{i_{1}} \bullet \cdots \bullet X_{i_{r}} & \text { if } \#\left(\bar{f}^{-1}(i)\right)=\#\left(\widehat{f}^{-1}(i)\right) \text { and } \bar{f}^{-1}(i)=\left\{P_{i_{1}}, \ldots, P_{i_{r}}\right\} .\end{cases}
$$

The $n$-fold coproduct of $X$ is defined by

$$
\Delta_{n}(X):=\sum_{I, \phi, f} \varepsilon_{(I, \phi, f)} h^{(I, \phi, f)} X_{(I, \phi, f)}^{(1)} \otimes \cdots \otimes X_{(I, \phi, f)}^{(n)},
$$

where

$$
\varepsilon_{(I, \phi, f)}=\prod_{\{(i, j) \in I \mid f(i, j)<f(\phi(i, j))\}} \varepsilon\left(a_{i, j} \cdot a_{\phi(i, j)}\right)
$$

and

$$
h^{(I, \phi, f)}=h^{(\# I-2 k+2 l) / 4} .
$$

Define $\Delta:=\Delta_{2}$. The following lemma yields the DG coalgebra on $A$ :

Lemma 16. Let $\Delta_{n}$ and $\Delta$ be defined as above. Then

(i) $\Delta_{n}$ is well-defined.

(ii) $\Delta$ is coassociative.

(iii) $b$ commutes with $\Delta$.

Proof. (i) To check that it is well-defined, one has to verify that if $\tilde{X} \in \tilde{B}$, then $\Delta_{n-1}(\tilde{X}) \in \sum_{i=1}^{n-1} i d^{\otimes i-1} \otimes \tilde{B} \otimes i d^{\otimes n-i}$. The proof of this is completely similar to $[23, \S 3.5]$ (by considering a quiver in [23] with just one vertex); we omit the details.

(ii) The proof is similar to $[23, \S 3.7]$. We have

$$
(\Delta \otimes i d) \circ \Delta=\Delta_{3}=(i d \otimes \Delta) \circ \Delta .
$$

As explained in $[23, \S 3.7]$, one can group the labelings 1 and 2 in $\Delta_{3}$ into labeling $1^{\prime}$ and consider $1^{\prime}$ and 3 ; this gives the first identity. Similarly, grouping the labelings 2 and 3 together into $2^{\prime}$ and considering 1 and $2^{\prime}$ gives the second identity.

(iii) The proof is by a direct verification similar to the proof for the DG Lie coalgebra. 
4.4. The Hopf identity. The proof is similar to [23, §3.8]. For any $X, Y \in A$,

$$
\begin{aligned}
\Delta(X Y) & =\Delta_{2}(X Y) \\
& =\sum_{\text {2-labelingsof } X Y}(X Y)^{\prime} \otimes(X Y)^{\prime \prime} \\
& =\sum_{\text {2-labelings of } X \text { and of } Y} X^{\prime} Y^{\prime} \otimes X^{\prime \prime} Y^{\prime \prime}+\sum_{\phi\left(I \cap P_{X}\right) \cap P_{Y} \neq \emptyset}(X Y)^{\prime} \otimes(X Y)^{\prime \prime} .
\end{aligned}
$$

The last summation is over all 2-labelings $(I, \phi, f)$ of $X Y$ such that $\phi\left(I \cap P_{X}\right) \cap P_{Y} \neq \emptyset$. However, in the product $X Y$, the heights of $Y$ are all greater than that of $X$, and if the set $\phi\left(I \cap P_{X}\right) \cap P_{Y}$ is nonempty, then by (56) and (57), one has

$$
\begin{aligned}
0 & =\sum_{(i, j) \in P_{X}} f(i, j)-f((i, j)+(0,1)) \\
& =\sum_{\left\{(i, j) \in I \cap P_{X} \mid \phi(i, j) \in P_{Y}\right\}} f(i, j)-f((i, j)+(0,1))<0,
\end{aligned}
$$

a contradiction. Hence, $\Delta(X Y)=\Delta(X) \Delta(Y)$.

Remark 17. The antipode map $S: A \rightarrow A$ is defined by replacing the heights $h_{i, j}$ in $X$ by $-h_{i, j}$, and then multiplying by $(-1)^{\#\left(P_{X}\right)}$. See $[23, \S 3.9]$.

4.5. Proof of the quantization. Let $\mathrm{B}_{C}$ be a basis for $C$. Let

$$
\mathrm{B}_{L}:=\left\{N\left(\left[a_{1}|\cdots| a_{n}\right]\right) \in L \mid a_{i} \in \mathrm{B}_{C} \text { for all } i\right\} .
$$

Then $\mathrm{B}_{L}$ is a basis for $L$. Let $S L$ be the symmetric algebra for $L$ and

$$
\mathrm{B}_{S L}:=\left\{x_{1} \bullet \cdots \bullet x_{k} \in S L \mid x_{i} \in \mathrm{B}_{L} \text { for all } i\right\} .
$$

Then $\mathrm{B}_{S L}$ is a basis for $S L$. Suppose $x \in \mathrm{B}_{S L}$ is the element

$$
N\left(\left[a_{1,1}|\cdots| a_{1, p_{1}}\right]\right) \bullet \cdots \bullet N\left(\left[a_{k, 1}|\cdots| a_{k, p_{k}}\right]\right),
$$

where $a_{i, j} \in \mathrm{B}_{C}$ for all $i, j$. Then we fix an element $Y(x) \in \tilde{A}$ of the form (35) where the sequence $h_{1,1}, \ldots, h_{k, p_{k}}$ is a permutation of $1,2, \ldots, \# P_{Y(x)}$. Let $\bar{Y}(x):=Y(x)+\tilde{B} \in A$.

Theorem 18. The set

$$
\mathrm{B}_{A}:=\left\{\bar{Y}(x) \in A \mid x \in \mathrm{B}_{S L}\right\}
$$

is a basis for A over $\mathbb{k}[h]$.

We refer the reader to [23, Corollary 4.2] for the proof of Theorem 18. (An alternative proof can also be given following the proof of the usual PBW Theorem for universal enveloping algebras of Lie algebras given, for example, in [5, §9.2].) It follows from Theorem 18 and the PBW Theorem for $L$ that $A$ is isomorphic to $U(L)[h]$ as $\mathbb{k}[h]$-modules. 
Note that any element of $L$ has a canonical lifting to an element of $A / h A$ since by (37) the heights do not matter. Thus, there is a natural map $L \rightarrow A / h A$. By (36), this map induces a homomorphism $\iota: U(L) \rightarrow A / h A$. It follows by Theorem 18 that $\iota$ is an isomorphism of DG Hopf algebras.

Let $x=N\left(\left[a_{1}|\cdots| a_{n}\right]\right) \in L$, and assume without loss of generality that its lifting $X \in A$ is represented by $N\left(\left[\left(a_{1}, 1\right)|\cdots|\left(a_{n}, n\right)\right]\right)$. From the definition of $\Delta$, we have

$$
\begin{aligned}
\Delta(X)= & 1 \otimes X+X \otimes 1 \\
& +h \sum_{i<j} \pm \varepsilon\left(a_{i} a_{j}\right) N\left(\left[\left(a_{1}, 1\right)|\cdots|\left(a_{i-1}, i-1\right)|\cdots|\left(a_{j+1}, j+1\right)|\cdots|\left(a_{n}, n\right)\right]\right) \\
& \otimes N\left(\left[\left(a_{i+1}, i+1\right)|\cdots|\left(a_{j-1}, j-1\right)\right]\right)+\text { higher order terms. }
\end{aligned}
$$

It follows that

$$
\delta(x) \equiv \frac{1}{h}\left(\Delta(X)-\Delta^{\mathrm{op}}(X)\right) \quad \bmod h
$$

4.6. Proof of Theorem 1. (i) If $(L, d)$ is a DG Lie algebra, then its universal enveloping $U(L)$ with the induced differential is a DG Hopf algebra; denote the induced differential by $b$. We have $H_{*}(U(L), b)$ is the enveloping algebra of $H_{*}(L, d)$ (see Quillen [22, Appendix, Proposition 2.1]). Therefore, by Theorems 9 and $15, H_{*}(A, b)$ quantizes the Lie bialgebra $H C_{*}(C)[\mathrm{m}-1]$.

(ii) This is immediate from Theorem 5 and Theorem 1 (i) .

Acknowledgements. The first author would like to thank Professor Yongbin Ruan for his encouragement during the preparation of this paper. The third author was partially supported by NSF grant DMS-0726154.

Open Access This article is distributed under the terms of the Creative Commons Attribution Noncommercial License which permits any noncommercial use, distribution, and reproduction in any medium, provided the original author(s) and source are credited.

\section{References}

1. Abbaspour, H., Tradler, T., Zeinalian, M.: Algebraic string bracket as a Poisson bracket. http://arxiv.org/ abs/0807.2351v3 [math.AT], 2008

2. Abbaspour, H., Zeinalian, M.: String bracket and flat connections. Alg. Geom. Top. 7, 197-231 (2007)

3. Andersen, J.E., Mattes, J., Reshetikhin, N.: The Poisson structure on the moduli space of flat connections and chord diagrams. Topology 35(4), 1069-1083 (1996)

4. Andersen, J.E., Mattes, J., Reshetikhin, N.: Quantization of the algebra of chord diagrams. Math. Proc. Camb. Phils. Soc. 124(3), 451-467 (1998)

5. Carter, R.: Lie Algebras of Finite and Affine Type. Cambridge: Cambridge Univ. Press, 2005

6. Cattaneo, A., Fröhlich, J., Pedrini, B.: Topological field theory interpretation of string topology. Commun. Math. Phys. 240(3), 397-421 (2003)

7. Cattaneo, A., Rossi, C.: Higher-dimensional $B F$ theories in the Batalin-Vilkovisky formalism: the BV action and generalized Wilson loops. Commun. Math. Phys. 221(3), 591-657 (2001)

8. Chas, M., Sullivan, D.: String topology, http://arXiv.org/abs/math/9911159v1 [math.GT], 1999

9. Chas, M., Sullivan, D.: Closed string operators in topology leading to Lie bialgebras and higher string algebra. In: The legacy of Niels Henrik Abel, Berlin: Springer, 2004, pp. 771-784

10. Chen, K.-T.: Iterated path integrals. Bull. AMS, 83(5), 831-879 (1977)

11. Cohen, R., Voronov, A.: Notes on string topology. In: String Topology and Cyclic Homology, Adv. Courses in Math: CRM, Barcelona, Basel-Boston: Birkhauser, pp. 1-95, 2006

12. Felix, Y., Thomas, J.-C.: Rational BV-algebra in String Topology. http://arXiv.org/abs/0705.4194v1 [math.AT], 2007 
13. Getzler, E., Jones, J.D.S., Petrack, S.: Differential forms on loop spaces and the cyclic bar complex. Topology 30, 339-371 (1991)

14. Ginzburg, V.: Non-commutative symplectic geometry, quiver varieties, and operads. Math. Res. Lett. 8(3), 377-400 (2001)

15. Goldman, W.: Invariant functions on Lie groups and Hamiltonian flows of surface group representations. Invent. Math. 85(2), 263-302 (1986)

16. Hamilton, A.: Noncommutative geometry and compactifications of the moduli space of curves. http:// arXiv.org/abs/0710.4603v1 [math.QA], 2007

17. Hamilton, A., Lazarev, A.: Symplectic $A_{\infty}$-algebras and string topology operations. http://arXiv.org/abs/ $0707.4003 \mathrm{v} 2$ [math.QA], 2007

18. Hess, K., Parent, P.-E., Scott, J.: CoHochschild homology of chain coalgebras. J. Pure Appl. Alg. 213(4), 536-556 (2009)

19. Jones, J.D.S.: Cyclic homology and equivariant homology. Invent. Math. 87, 403-423 (1987)

20. Lambrechts, P., Stanley, D.: Poincaré duality and commutative differential graded algebras. Ann. Sci. l'École Nor. Sup. 41, 495-509 (2008)

21. Loday, J.-S.: Cyclic homology. Second edition. Grundlehren der Mathematischen Wissenschaften, 301. Berlin: Springer-Verlag, 1998

22. Quillen, D.: Rational homotopy theory. Ann. of Math. 90(2), 205-295 (1969)

23. Schedler, T.: A Hopf algebra quantizing a necklace Lie algebra canonically associated to a quiver. Int. Math. Res. Notices 2005(12), 725-760 (2005)

24. Turaev, V.G.: Skein quantization of Poisson algebras of loops on surfaces. Ann. Sci. École Norm. Sup. (4) 24(6), 635-704 (1991)

Communicated by Y. Kawahigashi 